OPEN ACCESS

Edited by:

Michael Deyholos,

University of British Columbia,

Canada

Reviewed by:

Kashmir Singh,

Panjab University, Chandigarh, India

Dong Jiang,

Nanjing Agricultural University, China

*Correspondence:

Haifeng $\mathrm{LI}$

Ihf@nwsuaf.edu.cn

Specialty section:

This article was submitted to Plant Genetics and Genomics,

a section of the journal

Frontiers in Plant Science

Received: 02 November 2016 Accepted: 26 December 2016

Published: 10 January 2017

Citation:

Chen S and Li H (2017) Heat Stress Regulates the Expression of Genes at Transcriptional

and Post-Transcriptional Levels,

Revealed by RNA-sec

in Brachypodium distachyon.

Front. Plant Sci. 7:2067.

doi: 10.3389/fpls.2016.02067

\section{Heat Stress Regulates the Expression of Genes at Transcriptional and Post-Transcriptional Levels, Revealed by RNA-seq in Brachypodium distachyon}

\author{
Shoukun Chen ${ }^{1,2}$ and Haifeng Li ${ }^{1,2 *}$ \\ ${ }^{1}$ State Key Laboratory of Crop Stress Biology for Arid Areas, College of Agronomy, Northwest A\&F University, Yangling, \\ China, ${ }^{2}$ Xinjiang Agricultural Vocational Technical College, Changji, China
}

Heat stress greatly affects plant growth/development and influences the output of crops. With the increased occurrence of extreme high temperature, the negative influence on cereal products from heat stress becomes severer and severer. It is urgent to reveal the molecular mechanism in response to heat stress in plants. In this research, we used RNA-seq technology to identify differentially expressed genes (DEGs) in leaves of seedlings, leaves and inflorescences at heading stage of Brachypodium distachyon, one model plant of grasses. Results showed many genes in responding to heat stress. Of them, the expression level of 656 DEGs were altered in three groups of samples treated with high temperature. Gene ontology (GO) analysis showed that the highly enriched DEGs were responsible for heat stress and protein folding. According to KEGG pathway analysis, the DEGs were related mainly to photosynthesis-antenna proteins, the endoplasmic reticulum, and the spliceosome. Additionally, the expression level of 454 transcription factors belonging to 49 gene families was altered, as well as 1,973 splicing events occurred after treatment with high temperature. This research lays a foundation for characterizing the molecular mechanism of heat stress response and identifying key genes for those responses in plants. These findings also clearly show that heat stress regulates the expression of genes not only at transcriptional level, but also at post-transcriptional level.

Keywords: RNA-seq, heat stress, DEGs, Brachypodium distachyon, alternative splicing

\section{INTRODUCTION}

The natural environment for plants includes a set of abiotic and biotic stresses, and the responses to these stresses are complex (Cramer et al., 2011). Among the common abiotic stresses, high temperature stress is a major factor that influences plant growth and development (Rodriguez et al., 2015). Heat stress induces a series of physiological changes, such as an increased degree of lipid peroxidation, which decreases the thermal stability of the cell membrane (Mishkind et al., 2009; Narayanan et al., 2016); Blum et al. (2001) reported that the yield of wheat (Triticum aestivum) was 
positively correlated with the thermal stability of the cell membrane. Heat stress also produces a large number of freeradicals in chloroplasts and mitochondria, which can damage the membrane system of cells (Guan et al., 2013; Song et al., 2014). Additionally, the activity of radical-scavenging enzymes, such as superoxide dismutase (SOD), peroxidase (POD), and catalase (CAT) decreases and the content of malondialdehyde (MDA) increase. As a result of the release of free radicals, cells damage and even apoptosis can occur (Bita and Gerats, 2013; Hasanuzzaman et al., 2013).

The growth and development of some plant organs are affected by these responses to heat stress. For example, heat stress influences the development of inflorescences (Suzuki et al., 2014) and floral organs in cereal crops (Wu et al., 2015), especially the development of the male reproductive organs and the viability and germination of pollen grains (Young et al., 2004). The yield of these crop plants also is affected. A crop simulation model demonstrated that a variation of $2^{\circ} \mathrm{C}$ in average growing-season temperatures could lead to up to $50 \%$ yield reductions in grain production in wheat (Asseng et al., 2011). Additionally, during the filling stage in wheat, high temperature can influence the accumulation of organic matter and starch (Stratonovitch and Semenov, 2015). In general, high temperature could lead to catastrophic losses in cereal crops (Bita and Gerats, 2013).

Brachypodium distachyon is an important model plant for studying grasses (Draper et al., 2001), as it has a fully sequenced genome (International Brachypodium Initiative, 2010). It is an specially important model because the $B$. distachyon genome is close to wheat and its relatives (Subburaj et al., 2015). Previous studies have shown that high temperature results in several aspects in B. distachyon. First, it influences the growth and reduces the tiller numbers. Second, it disrupts the development of anthers and pollen (Harsant et al., 2013); high temperature had an effect on anther length and dehiscence, as well as the development of pollen. Additionally, results show that with the increase in daytime temperature, the number of pollen grains decreases, and when the temperature reaches $36^{\circ} \mathrm{C}$, most pollen development is arrested at the uninucleate stage. In addition to the effect on pollen development, pollen viability, and deposition, the germination on the stigma is also affected by high temperature (Harsant et al., 2013). As a result, the setting rate and the harvest index of $B$. distachyon decreased under heat stress.

With the increased occurrence of extreme heat events, the effects on plant growth and development are becoming a severe issue for agriculture. It is imperative to identify the molecular mechanism that drives the response to heat stress. Previous studies have shown that the plant response to high temperature is associated with multiple processes and mechanisms involving heat shock proteins (HSPs), transcription factors (TFs), and other stress related genes (Qin et al., 2008; Liu et al., 2012; Hasanuzzaman et al., 2013), as well as changes in protein domains, phylogenetic relationships, and gene and protein structures (Cao J. et al., 2016). Recent studies in $B$. distachyon found that some genes are regulated by heat, such as 14-3-3 gene family (Cao H. et al., 2016), PP2C gene family (Cao J. et al., 2016), TIFY family genes (Zhang et al., 2015), NAC gene family (You et al., 2015) and bZIP TFs (Liu and Chu, 2015). High-throughput sequencing is an accessible and widely used technology that allows the sequencing of an entire transcriptome or genome in a timely and cost-effective manner (Reon and Dutta, 2016). Transcriptome studies of heat stress effects on rice, wheat, tomato, and grape have been reported (Frank et al., 2009; Liu et al., 2012; Kumar et al., 2015a; Wu et al., 2015). Studies in rice found that heat responsive genes in rice panicles (Zhang et al., 2012) and flag leaves (Zhang et al., 2013) were mainly involved in transcriptional regulation, transport, protein binding, and antioxidant and stress responses. Frank et al. (2009) found that HSPs, reactive oxygen species (ROS) scavengers, hormones, and sugars were involved in responses to heat stress in maturing tomato (Solanum lycopersicum) microspores. Liu et al. (2012) reported that the number of heat stress-regulated genes was almost twice the number of recovery-regulated genes, and that these heat stress-related transcriptional changes regulate a large number of important traits and biological pathways in grape (Vitis vinifera L.). In wheat, the combination of heat and drought stress acted in a synergistic manner rather than an additive manner (Liu et al., 2015). The regulatory network analysis of heat shock transcription factors (HSFs) and dehydration responsive element binding (DREBs) suggest that they were involved in the regulation of drought stress, heat stress, and combined heatdrought stress responses.

Since high temperature resulted in abnormal development of anthers, pollen, and setting rate, and harvest index, we were interested in investigating key genes responsible for resistance to heat stress. We used RNA-Seq to characterize differentially expressed genes (DEGs) not only in leaves of seedlings, but also in leaves and inflorescences of $B$. distachyon at the heading stage. We found that the heat stress not only regulates the expression level of genes, but also regulates alternative splicing (AS) of genes. Our findings lay a foundation for understanding the molecular mechanisms involved in response to heat stress.

\section{MATERIALS AND METHODS}

\section{Plant Materials, Growth Conditions, and Treatments}

Brachypodium distachyon Bd-21 was grown in an artificial climate chamber at $26 / 22^{\circ} \mathrm{C}$ (day/night) with a photoperiod of 16/8 h (day/night). Two-week-old seedlings and eight-weekold plants at the heading stage were selected for heat stress treatment that was applied after plants were transferred to a growth chamber at $42^{\circ} \mathrm{C}$. Seedling leaves were collected at $0 \mathrm{~h}(\mathrm{CK} 1)$ and $5 \mathrm{~h}(\mathrm{~S} 1)$, heading-stage leaves were collected at $0 \mathrm{~h}(\mathrm{CK} 2)$ and $1 \mathrm{~h}$ (S2), and inflorescences were collected at $0 \mathrm{~h}(\mathrm{CK} 3)$ and $1 \mathrm{~h}(\mathrm{~S} 3)$ after heat treatment, respectively. After collection, samples were frozen immediately in the liquid nitrogen and stored at $-80^{\circ} \mathrm{C}$ until further use. Every sample had two biological replicates that were sequenced independently. 


\section{RNA Extraction, Libraries Construction, Transcriptome Sequencing and Mapping}

Total RNA was extracted using a TRIZOL kit (Sangon Biotech, Shanghai, China) according to the manufacturer's protocol and treated with DNaseI (Sangon Biotech, Shanghai, China). Sequencing libraries were prepared with the Ion Total RNASeq Kit v2 according to the protocol (Life technologies, USA). Emulsion PCR was performed using the cDNA library as a template. The Template Positive Ion PI ${ }^{\mathrm{TM}}$ Ion Sphere ${ }^{\mathrm{TM}}$ Particles were enriched and loaded on the Ion $\mathrm{PI}^{\mathrm{TM}}$ chip for sequencing. In total, 12 samples were sequenced.

The online bioinformatics program, FAST- $\mathrm{QC}^{1}$, was used to evaluate the quality of the sequencing data. After quality testing, clean reads were mapped to the reference genome of $B$. distachyon with Mapsplice software (Wang K. et al., 2010), in which the core Bowtie program identified the exon-exon splicing accurately.

\section{Identification of Alternative Splicing}

After mapping, an 'accepted_hits.bam' file was generated that included information regarding the chromosome position for exonic reads and exon-exon junction reads. All junction reads had to meet two criteria: (i) a read must match perfectly to each of the two flanking regions of a potential junction site with more than 6-nt; and (ii) a junction site that has $>3$ non-redundant reads in both the $\mathrm{CK}$ and $\mathrm{S}$ samples must be filtered. Afterward, an ASD (Alternative Splicing detector) (Zhou et al., 2014) was developed to fulfill the following tasks: (a) reconstruction of exon-clusters based on the aforementioned reannotated transcriptome for identifying modes of AS events for each exon-cluster; (b) count the number of junction reads that align either to the inclusion or exclusion isoforms in both the $\mathrm{CK}$ and $\mathrm{S}$ samples, and calculate the $P$-value using junction read-counts between $\mathrm{CK}$ and $\mathrm{S}$ samples by the Fisher exact test; (c) calculate read coverage for the alternative exons and their corresponding genes in both CK and S samples, and calculate a second $P$-value by the Fisher exact test based on the alternative exon read coverage relative to the gene read coverage between $\mathrm{CK}$ and S samples; and (d) combine the two $P$-values to get an adjusted $P$-value using a weighted arithmetic equation for assessing the statistical difference in AS between CK and S sample groups.

\section{Identification of DEGs}

With a $\log$-fold expression change of $\log 2 \mathrm{FC}>1$ or $<-1$, and a threshold of false discovery rates (FDR $<0.05)$, DEGs were filtered using a DEGSeq algorithm (Wang L. et al., 2010).

\section{GO Analysis}

Gene ontology (GO) was applied to analyze the main functions of DEGs according to the GO database and to determine the biological implications of unique genes in the significant or representative profiles (Ashburner et al., 2000). The GO annotations from $\mathrm{NCBI}^{2}$, UniProt ${ }^{3}$, and Gene

\footnotetext{
${ }^{1}$ http://www.bioinformatics.babraham.ac.uk/projects/fastqc

${ }^{2}$ http://www.ncbi.nlm.nih.gov

${ }^{3}$ http://www.uniprot.org/
}

Ontology ${ }^{4}$ were downloaded Fisher's exaction and $\chi^{2}$ tests were used to classify the GO categories with a FDR (Dupuy et al., 2007) calculated to correct the $P$-value. The smaller the FDR, the smaller the error there was in judging the $P$-value.

\section{Pathway Analysis}

The identification of significant pathways for DEGs was according to the Kyoto Encyclopedia of Genes and Genomes (KEGG) database ${ }^{5}$. A Fisher exaction test was used to screen the significant enrichment pathways with a threshold of significance defined by $P$-value and FDR. The resulting $P$-values were adjusted using the FDR algorithm. Then, pathway categories with FDRs $<0.05$ were reported. Enrichment provides a measurement of the significance of the genes function: as the enrichment increases, the corresponding function is more specific. This helped us identify more significant pathways from our DEG results.

\section{Identification of TFs}

The B. distachyon TF database PlantTFDB $3.0^{6}$ (Jin et al., 2014), was used as a reference TF database. Putative TFs in B. distachyon were identified using BLASTx with a cut-off $E$-value of $1 \times 10^{-5}$ (Kalra et al., 2013).

\section{Validation of Gene Expression}

To validate the RNA-Seq data, the expression of selected upor down-regulated genes, and alternative splicing events were confirmed by quantitative reverse transcription-polymerase chain reaction (qRT-PCR) or semi-quantitative RT-PCR analysis. qRT-PCR reactions were carried out as described previously (Liu et al., 2016) and every reaction was performed in triplicate. Data acquisition and analysis were performed using the QuantStudio ${ }^{\mathrm{TM}}$ Real-Time PCR Software (ThermoFisher Scientific). Samples were normalized using ACTIN gene expression (Hong et al., 2008) and relative expression levels were determined using the $2(-\Delta \Delta \mathrm{Ct})$ analysis method (Livak and Schmittgen, 2001).

For semi-quantitative RT-PCR analysis, the concentration of template was first normalized using ACTIN expression (Hong et al., 2008). Subsequently, $15-\mu l$ reactions containing $7.5 \mu \mathrm{l}$ $2 \times$ Taq MasterMix (ComWin Biotech, Beijing), $0.75 \mu$ l of each forward and reverse primer, $0.5 \mu \mathrm{l} \mathrm{cDNA}(5.0 \mathrm{ng} / \mu \mathrm{l})$, and $5.5 \mu \mathrm{l}$ ddH2O. PCR cycling conditions began with $94^{\circ} \mathrm{C}$ for $5 \mathrm{~min}$, and were followed by 40 cycles of $94^{\circ} \mathrm{C}$ for $30 \mathrm{~s}, 58-64^{\circ} \mathrm{C}$ for $30 \mathrm{~s}, 72^{\circ} \mathrm{C}$ for $30 \mathrm{~s}$, and $72^{\circ} \mathrm{C}$ for $10 \mathrm{~min}$.

\section{RESULTS}

\section{Quality of RNA-seq Data}

To characterize DEGs responsive to heat stress in B. distachyon, we performed RNA-Seq on twelve samples using the Ion

\footnotetext{
${ }^{4}$ http://www.geneontology.org/

${ }^{5} \mathrm{http}: / /$ www.kegg.jp/

${ }^{6} \mathrm{http}: / /$ planttfdb.cbi.pku.edu.cn/download.php
} 
Proton $^{\text {TM }}$ System. For each transcriptome, a total of 13.4-17.1 million clean reads were collected. Since the size of the $B$. distachyon genome is about $272-\mathrm{MB}$, and the poly(A)-tail mRNA size should be about 2.6-5.2-MB (1-2\% of genome size). In the current study, the data from each $B$. distachyon sample were more than 2-GB, which could cover the poly(A)-tail mRNA for 384 to 770 times. Previously, 6,399 genes were reported to be involved in abiotic stress (Priest et al., 2014). Among these genes, 6,320, 6,320 and 6,330 genes were detected in our S1, S2, and S3 samples, respective. These results indicate that our data were sufficient for further analysis. Evaluation of the data quality showed that the length and the GC content of the reads were in accordance with the criteria (ReadsFilter > 90\%, GC > 48\%) (Supplementary Table S1). Additionally, more than $93 \%$ of the filtered reads mapped to the annotated reference genome (Supplementary Table S1). The raw data were submitted to the NCBI database under the SRA accession number: SRS1792875.

\section{Identification and Validation of DEGs}

To identify DEGs, we used the RPKM method (Reads per KB per Million reads) to calculate the expression levels of the unigenes. There were 3,524 (1,459 up-regulated, 2,065 downregulated), 3,787 (2,154 up-regulated, 1,633 down-regulated) and 2,081 (1,465 up-regulated, 616 down-regulated) DEGs characterized in our three groups, respectively. In total, 6,609 DEGs were identified and of them, the expression of 656 (510 up-regulated genes and 114 down-regulated) genes were altered in all three groups of sample groups simultaneously (Figure 1). To verify the expression level of the DEGs, RTqPCR was conducted, and the expression level of 13 randomly selected genes was analyzed. Results show that the expression trends were consistent with the RNA-Seq data (Figure 2, Supplementary Table S2).

Although only a few $B$. distachyon genes were reported to function in heat stress resistance, several genes from wheat, rice, and maize are thought to be responsible for resistance to heat stress. Therefore, we focused our analysis on the expression of their homologues in B. distachyon.

In rice, $D R E B 2 B$ encodes a TF that when it was overexpressed in Arabidopsis, the transgenic plants display an enhanced resistance to heat stress (Matsukura et al., 2010). In our sequencing data, the expression level of the homolog in $B$. distachyon (Bradi2g29960) for the rice DREB2B and maize $Z m D R E B 2 A$ genes was up-regulated in S1, S2, and S3. The expression of TaMBF1c, which encodes a multiprotein bridging factor, is induced by heat stress and confers heat tolerance in transgenic rice (Qin et al., 2015). The expression of BdMBF1c was up-regulated $80.211,2751.3$, and 358.86757 times in S1, S2, and S3, compared with control, respectively, Another wheat gene, TaHsfA6f, was reported to activate the expression of its downstream genes that are responsible for heat tolerance (Xue et al., 2015). In our data, the expression of the TaHsfA6f homologue in B. distachyon was elevated by 1.2476, 11.2309, and 99.564263 times in S1, S2, and S3, respectively, compared with that in the control. These results indicated that the

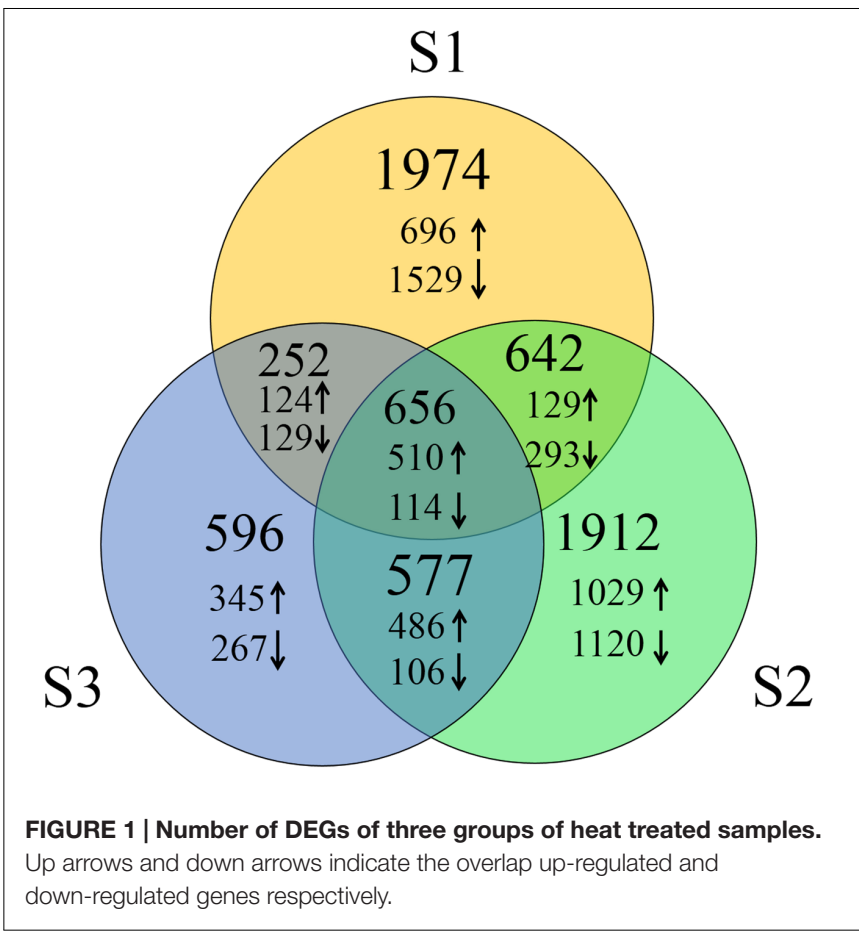

identified DEGs included genes responsible for responding to heat stress.

\section{Hierarchical Clustering Analysis and Functional Enrichment Analysis of the DEGs}

Genes with similar patterns of expression can often have overlapping biological functions, and these similar genes may share functionality or participate in the same biological processes (BP; Huang et al., 2015). We used the RPKM expression values to analyze gene expression and perform a hierarchical clustering analysis of the gene expression patterns in 656 DEGs using HemI 1.0 (Deng et al., 2014) and the results are shown in Figure 3.

Gene ontology assignments were used to predict the functions of $B$. distachyon unigenes by classifying them by various BPs. Genes were classified into categories with three independent ontologies including biological process, molecular function (MF), and cellular components (Ashburner et al., 2000). Based on the results of the unigene annotation and GO analysis, 3,221, 3,389 , and 1,890 unigenes were annotated to $1,763,2,699$, and 1,392 significant biological process GO terms $(p<0.05)$. Among these BPs, genes highly enriched in S2 and S3 were GO:0009408 (response to heat), GO:0006457 (protein folding), GO:0042542 (response to hydrogen peroxide), and GO:0009644 (response to high light intensity); while GO:0010200 (response to chitin) and GO:0015979 (photosynthesis) were highly enriched in S1 (Figure 4). Moreover, the cellular components category consisted of 330, 329, and $227 \mathrm{GO}$ terms $(p<0.05)$ in S1, S2, and S3, respectively. Among them, GO:0009579 (thylakoid), GO:0009535 (chloroplast thylakoid membrane), GO:0009522 (photosystem I), and GO:0009523 (photosystem II) were highly enriched in S1 


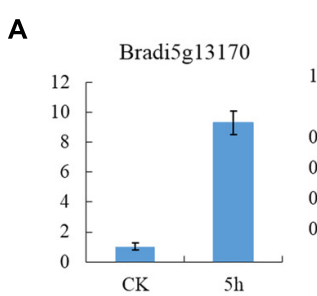

B

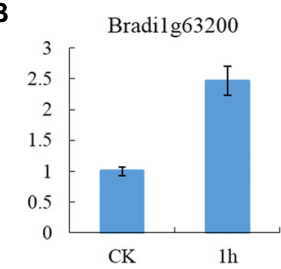

C

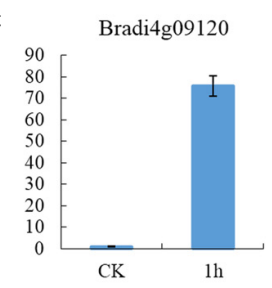

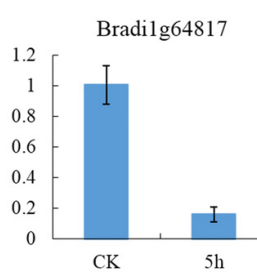

Bradi3g47640

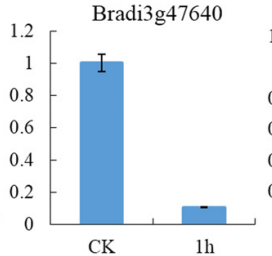

CK 1 h

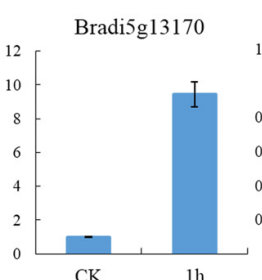

CK

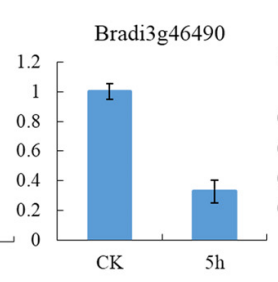

Bradi2g11100

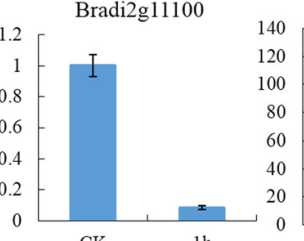

CK $\quad$ lb

Bradi4g25900

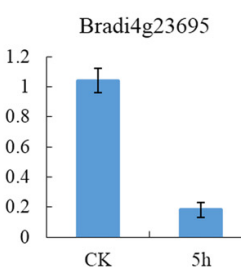

Bradi3g57530

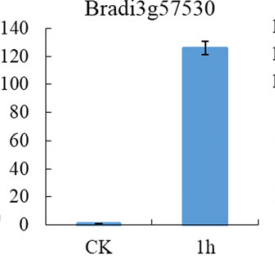

CK 1b

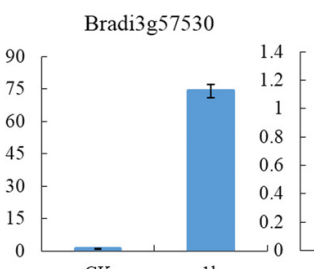

CK
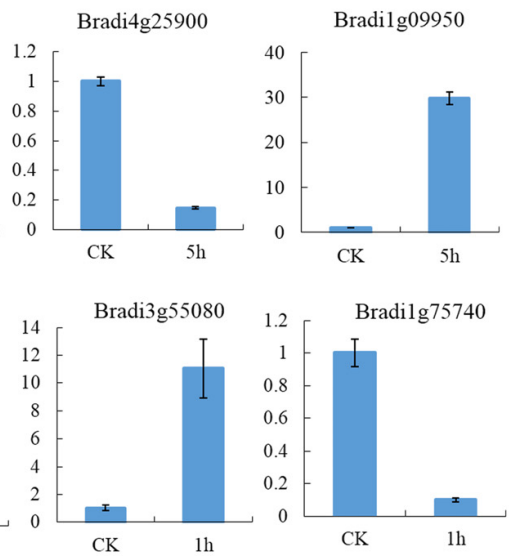

CK $1 \mathrm{~h}$

Bradilg64817

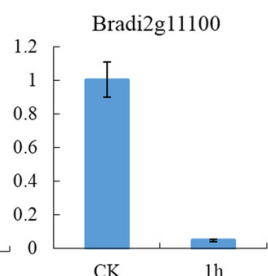

$1 \mathrm{~h}$

FIGURE 2 | Expression profile of randomly selected DEGs. (A) Relative expression level of six genes in leaves of seedlings, (B) Relative expression level of six genes in leaves and $\mathbf{( C )}$ inflorescences at heading-stage, respectively.

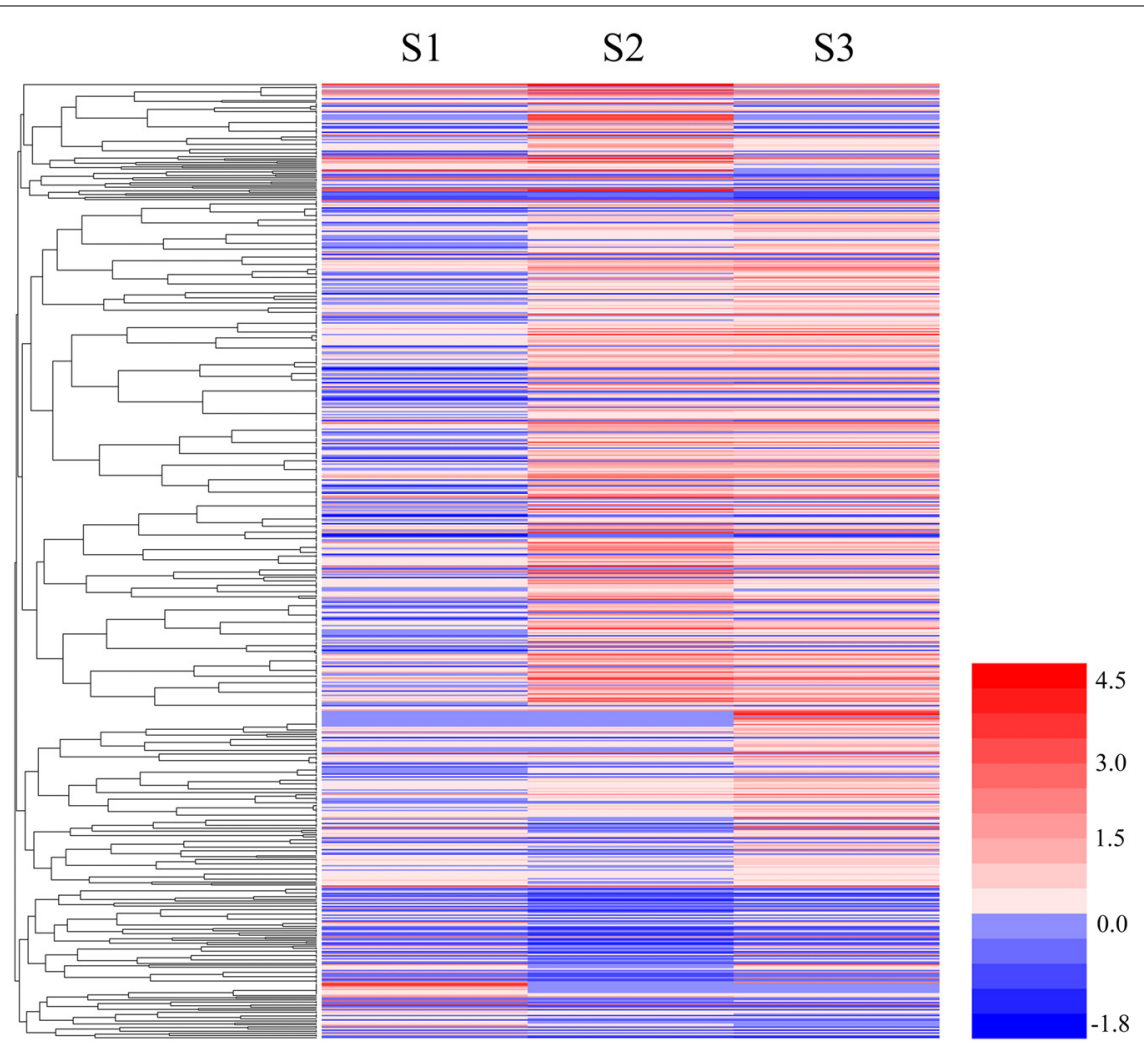

FIGURE 3 | Cluster analyses of DEGs among S1, S2, and S3. The color key represents the value of log10 (RPKM). Red represents highly expressed (up-regulated) genes, while blue represents down-regulated genes. 


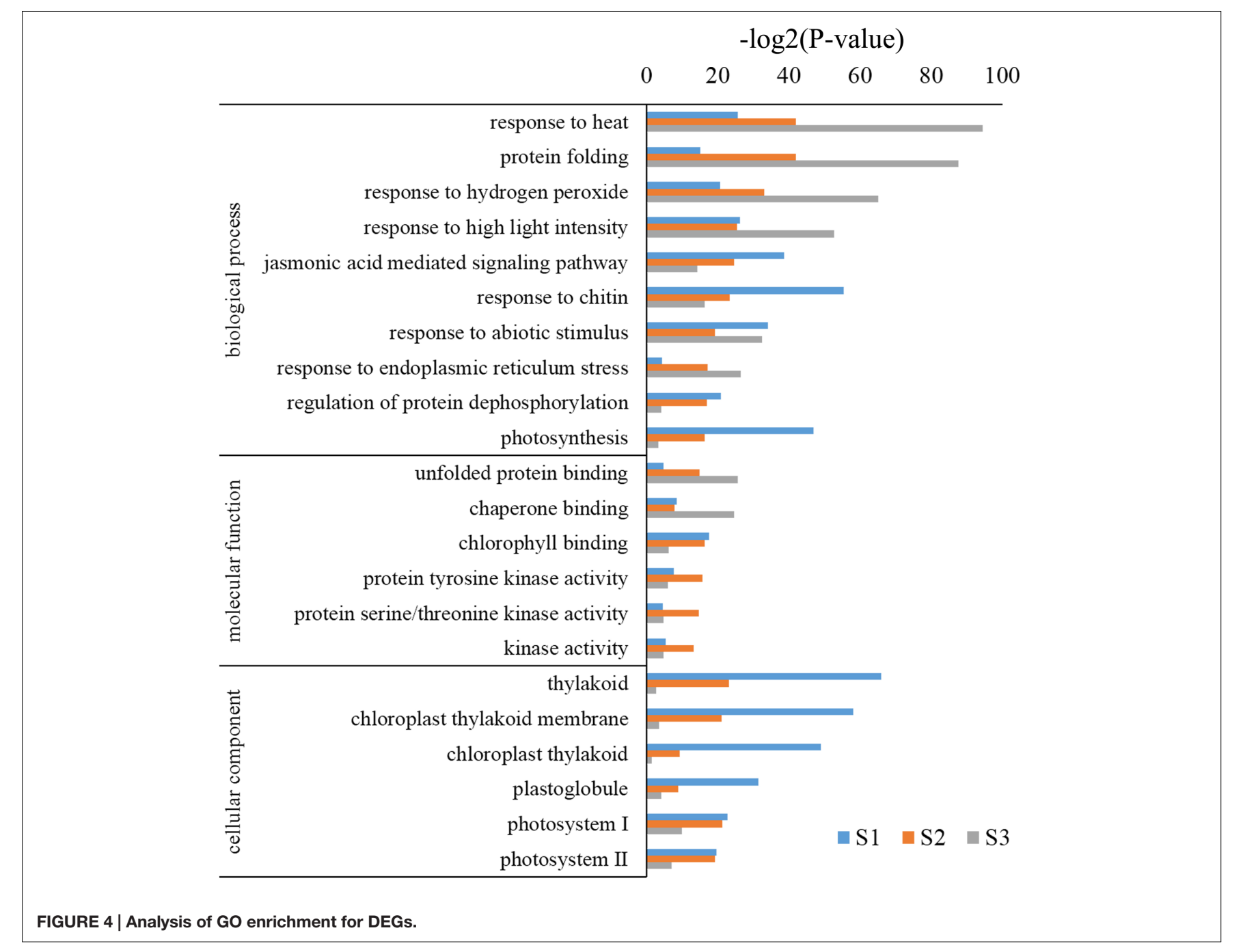

and S2, but not in S3 (Figure 4). MFs consisting of 1,149, 1,027 , and $798 \mathrm{GO}$ terms $(p<0.05)$ and highly enriched terms included GO:0016168 (chlorophyll binding) in S1 and S2, and GO:0051087 (chaperone binding) and GO:0051082 (unfolded protein binding) in S3 (Figure 4). These results suggest that different types of genes respond to heat stress at different developmental stages in B. distachyon.

KEGG is a database of biological systems and resource that integrate genomic, chemical, and systemic functional information (Kanehisa et al., 2012). All DEGs were screened in the KEGG database for pathway annotation and the significance level was calculated using the Fisher's exact test $(p<0.05)$. In total, $23.60 \%(760 / 3,221), 20.51 \%(695 / 3,389)$ and $21.11 \%$ $(399 / 1,890)$ of the differentially expressed unigenes in S1, S2, and S3 samples were annotated, respectively. There were 31,18 , and 16 pathway categories found to be significantly enriched in each developmental stage, as well. As shown in Supplementary Table S3, in S1 and S2, the most significantly enriched genes were antenna proteins (PATH:00196) involved in the photosynthetic pathway. In S3, the most significantly enriched genes were involved in protein processing pathways in the endoplasmic reticulum (PATH:04141). Additionally, genes encoding components of the spliceosome (PATH:03040) were expressed in S1 and S3 at high levels, and genes associated with photosynthesis, including PATH:00196 (Photosynthesis-antenna proteins), PATH:01200 (Carbon metabolism), PATH:00710 (Carbon fixation in photosynthetic organisms) and PATH:00195 (Photosynthesis) were also highly expressed. These results are consistent with the fact that leaves are the main organs to conduct photosynthetic reactions.

\section{Identification and Verification of Differentially Expressed Transcription Factors}

Transcription factors are important regulators that participate in the response to biotic and abiotic stresses (Lindemose et al., 2013). In order to better understand the molecular mechanism regulating the response to heat stress in B. distachyon, 454 TFs were identified from DEGs according to the rules of family assignment illustrated in PlantTFDB (Guo et al., 2008). These TFs belong to 49 TF families, such as ERF (42), bHLH (33), bZIP (33), 


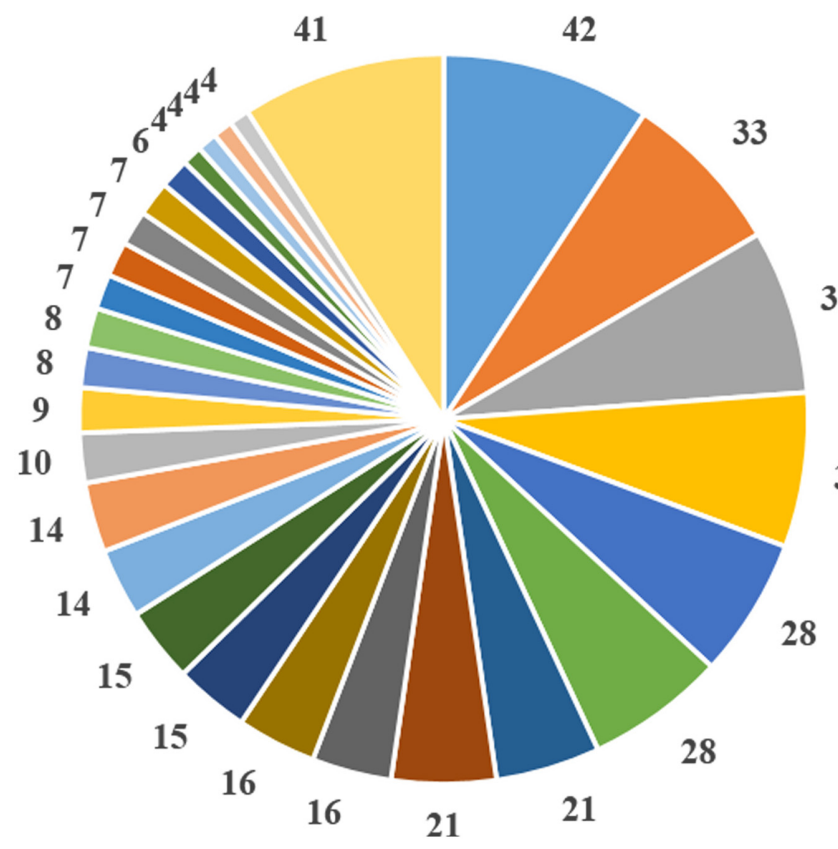

\begin{tabular}{|c|c|}
\hline - ERF & — bHLH \\
\hline bZIP & MYB \\
\hline - NAC & " WRKY \\
\hline — $\mathrm{C} 2 \mathrm{H} 2$ & - MYB_related \\
\hline - $\mathrm{C} 3 \mathrm{H}$ & a G2-like \\
\hline - GRAS & 匹 HSF \\
\hline FAR1 & - HD-ZIP \\
\hline CO-like & Trihelix \\
\hline GATA & TCP \\
\hline ARF & - GeBP \\
\hline SBP & TALE \\
\hline - Dof & - AP2 \\
\hline - GRF & M-type \\
\hline ZF-HD & Others \\
\hline
\end{tabular}

FIGURE 5 | Distribution of differentially expressed transcription factors in gene families.

MYB (31), NAC (28), and WRKY (28) (Figure 5). Compared with previously results (You et al., 2015), many NAC genes in S1 and S2 samples showed consistent trends in expression. For example, the expression of BdNAC006 (Bradilg17440), BdNAC034 (Bradi2g24987), BdNAC042 (Bradi2g53260), and BdNAC062 (Bradi3g47627) was up-regulated by heat stress both in 3-week-old heat-treated seedlings (You et al., 2015) and in our S1 and S2 samples (Supplementary Table S4). Similarly, the expression of BdNAC009 (Bradilg29857), BdNAC016 (Bradilg50057), BdNAC025 (Bradilg76207), BdNAC033 (Bradi2g24790), and BdNAC043 (Bradi2g55780) was downregulated in both 3 -week-old heat-treated seedlings (You et al., 2015) and S1 and S2 samples (Supplementary Table S6). These results suggest that these NAC genes are involved in heat stress response. We selected 10 interesting TFs including three ERFs (Bradi2g09434, Bradi2g29960, Bradi3g51630), three HSFs (Bradilg05550, Bradi3g26920, Bradi4g35780), two MYBs (Bradi4g04627, Bradi5g15760), and two NACs (Bradilg29857, Bradi2g53260) for further validation. Results showed that the expression level of these genes was consistent with the RNA-Seq data (Figure 6).

\section{Identification of Alternative Splicing Events}

Alternative splicing (AS) is a widespread co- and posttranscriptional regulatory process in plants. It can produce more than one isoform of mRNA transcripts and proteins from one gene (Stamm et al., 2005; Labadorf et al., 2010). AS is involved in most plant processes and is particularly prevalent in plants exposed to environmental stresses (Macaulay et al., 2015;
Mandadi and Scholthof, 2015). To investigate the influence of heat on AS, we analyzed the events of AS with a threshold of false discovery rates (FDR < 0.05). In total, 1,973 $(604,1,082$, and 287 events in S1, S2, S3, respectively) AS events were identified distributed across 451 DEGs, including 131, 320, 110 in S1, S2, S3 sample groups, respectively (Supplementary Table S5). Cassettes A3SS, A5SS, and RI are the most predominant AS types under heat stress (Supplementary Figure S1A). Of the 451 DEGs, 18 generated isoforms were identified in the three groups of samples after heat treatment (Supplementary Figure S1B). These AS genes were highly enriched for RNA splicing and protein folding BPs in GO enrichment analysis, while the spliceosome was most highly enriched in the KEGG pathway (Supplementary Figures S1C,D).

To verify the AS events, the two genes Bradi4g16980 and Bradi3g07286 were selected for further analysis. The AS of Bradi4g16980 can occur in a skipped exon manner, indicated by the Integrative Genomics Viewer (IGV) map of RNASeq reads (Figure 7A). According to the predicted sites and the length of exons, primers were designed and semi-quantitative RT-PCR experiments were performed. As shown in Figures $\mathbf{7 B}, \mathbf{C}$, in addition to bands $a$ that included exons $10,11,12$, and 13 , and $b$ that included exons 10, 11, and 13 that could be amplified in leaves of seedlings under normal conditions, band $c$, which included exons 10 and 13, was amplified in heat-treated samples. This result indicated another isoform was formed during the RNA processing by skipping the 12th exon. Similarly, in Bradi3g07286, the data show the occurrence of AS (Figure 8A). The PCR products from leaves at the heading stage only contained 
A

c
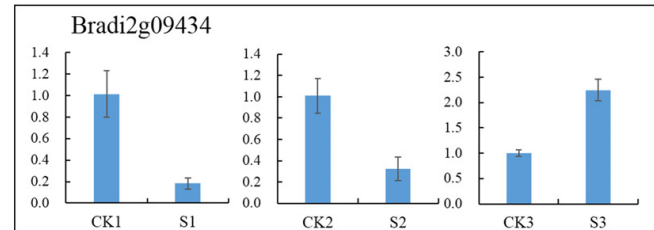

Bradi2g29960
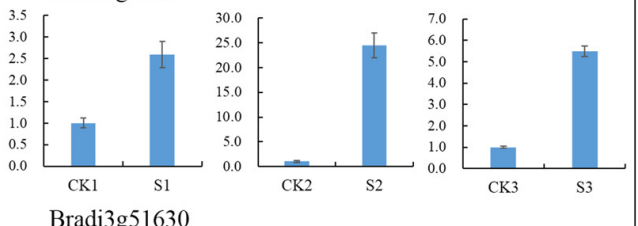

Bradi3g51630
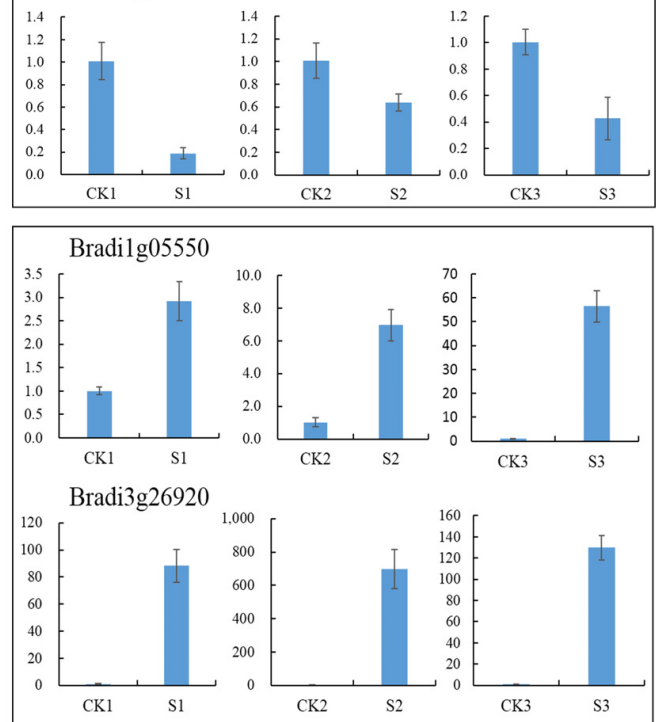

Bradi4g35780
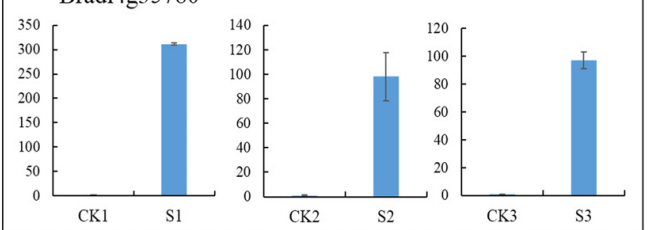

B
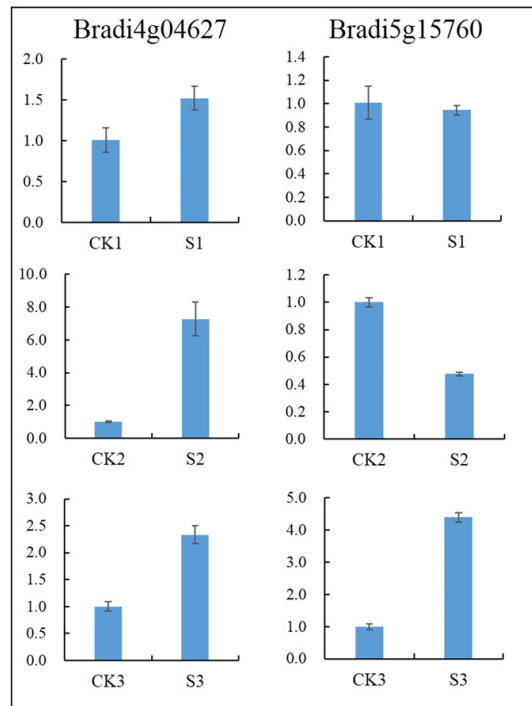

D
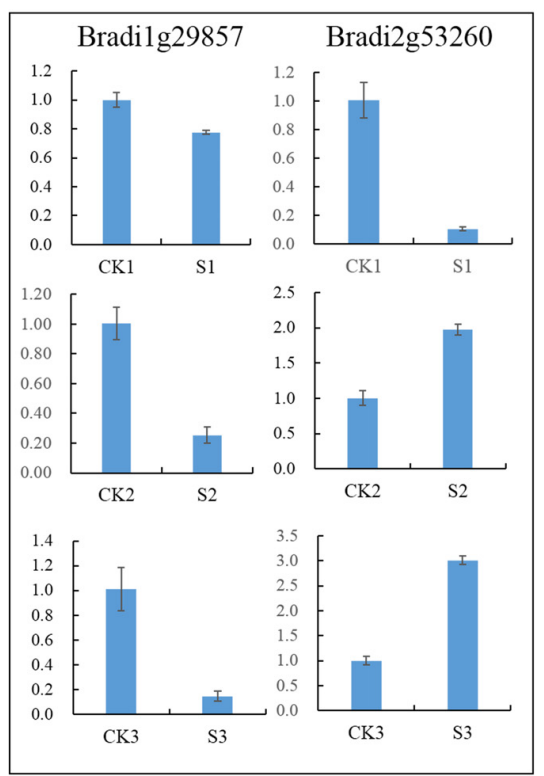

FIGURE 6 | Relative expression of selected TF genes. (A) ERF genes, (B) MYB genes, (C) HSF genes and (D) NAC genes.

one band; however, after heat treatment, another two bands emerged (Figure 8B), indicating that heat stress induced AS. Analysis of the genome sequence suggested that band $b$ product excluded the 10th exon that was included in band $a$ product; while band $c$ excluded both 10 th and 11 th exons (Figure 8C).

\section{DISCUSSION}

\section{The Influence of Heat Stress on Plants Is Complicated}

High temperature and drought are perhaps the two most serious environmental factors that limit crop growth and yield worldwide, and the combination of these stresses can cause many physiological changes that affect crop yield and quality (Rizhsky et al., 2002, 2004; Mittler, 2006; Prasad et al., 2011; Vile et al., 2012), especially at the reproductive stage. In barley, heat stress significantly increases the number of aborted spikes and decreases kernel weight (Rollins et al., 2013). Heat stress causes a significant decrease in grain number, spikelet fertility, grain yield and harvest index as well as chlorophyll content (Prasad et al., 2011). In maize and spring wheat, heat stress decreases pollen fertility and grain number (Westgate, 1994; Prasad et al., 2011). Additionally, heat stress affects a series of physiological traits in many plants. For example, it inhibits photosynthesis mainly via altering non-stomatal traits, such as electron transport capacity 


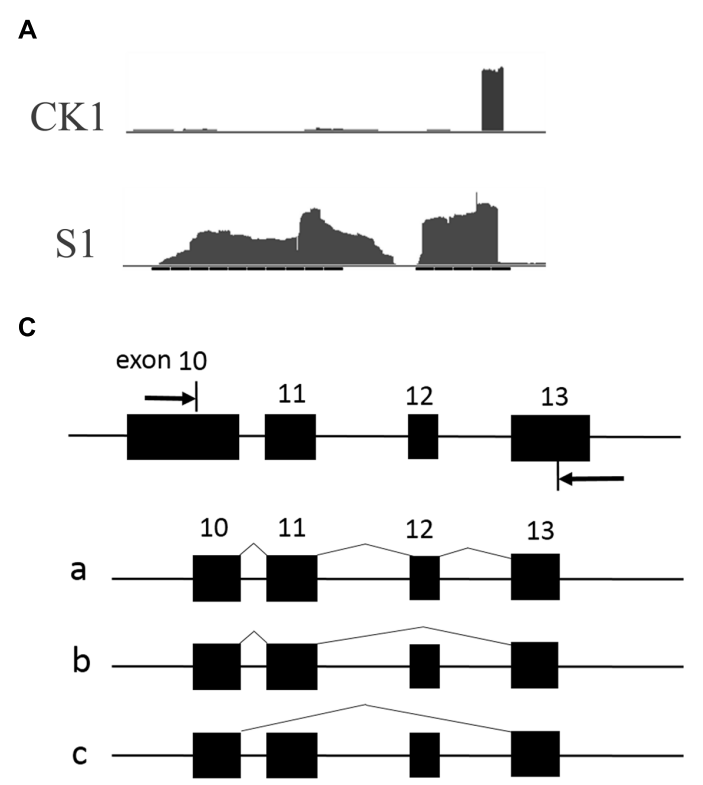

B $\quad$ Bradi4g 16980

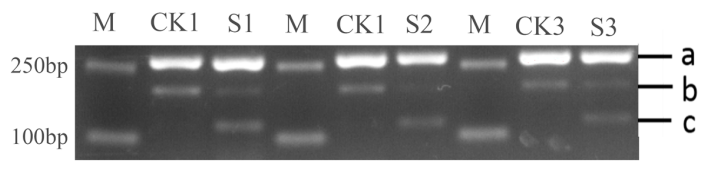

Actin

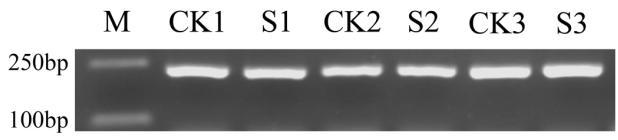

FIGURE 7 | Alternative splicing events in Bradi4g16980. (A) The IGV map of RNA-seq reads. The reads showed that the middle exon was differentially spliced. (B) Result of semi-quantitative RT-PCR. (C) Diagram of isoforms a, b, and c. Arrows in (C) indicate primers.

and Rubisco activity (Salvucci and Crafts-Brandner, 2004a,b; Way and Oren, 2010). In cotton, the photosynthetic rate and stomatal conductance decreases and leaf temperatures increase under heat stress (Carmo-Silva et al., 2012). These observations demonstrate the complicated influence of high temperature on plants.

At the molecular level, the expression levels of more than one-fourth of the genes in the $B$. distachyon genome $(6,609 / 25,532)$ were altered after heat treatment (Figure 1). These genes are involved in cell membranes, chloroplasts, the endoplasmic reticulum, and other organelles. Proteins encoded by these DEGs play multiple roles and participate in constituting spliceosomes, photosystem I, photosystem II, and so on. In addition to involvement in the response to heat and other abiotic stresses like hydrogen peroxide and chitin, the result of GO enrichment analysis showed that these DEGs were involved in 12 other BPs such as photosynthesis, protein dephosphorylation, and protein folding (Figure 4). The processes of photosynthesis, protein processing, and mRNA splicing were especially present in this gene analysis. GO enrichment analysis indicated that in response to heat; most of the up-regulated genes were involved in protein folding, protein processing, the spliceosome, and some physiological metabolic pathways. The GO terms related to photosynthesis were enriched in genes that were down-regulated in response to high temperature. Of the 6,609 DEGs, 454 genes encoded different types of TFs that regulate plant development and growth, and the response to biotic and abiotic stresses by activating or repressing the expression of their target genes. These results are similar to what has been shown in rice and bread wheat, where the expression levels of a majority of genes are changed under heat stress.
Additionally, some reports show that the expression profiles of miRNAs involved in plant growth and development are altered under high temperature (Xin et al., 2010; Kumar et al., 2015b). The altered expression of miRNAs has been implicated in the effects on plant growth and development during environmental stress (Ding et al., 2013). Recently, Kumar et al. (2015b) proposed that the expression of heat-responsive miRNAs was induced by elevated temperature, which in turn repressed the expression of their target genes and TFs. Taken together, previous work and our study suggest that the heat stress affects many BPs and pathways by altering the expression of genes that encode TFs and miRNAs.

\section{Heat Stress Induces Alternative Splicing}

Alternative splice sites are used to generate two or more mRNA product from one pre-mRNA transcript (Staiger and Brown, 2013) to improve the diversity of functional proteins (Sablok et al., 2011). High throughput sequencing for transcript profiling in plants has revealed that AS affects a much higher proportion of the transcriptome than originally predicted. RNASeq technologies have greatly facilitated the comprehensive survey of AS events (Sablok et al., 2011; Walters et al., 2013). AS is very popular in plants and the frequency of AS in Arabidopsis for example, is more than $61 \%$ of intron-containing genes under normal growth conditions (Marquez et al., 2012); and in B. distachyon, AS is also common (Sablok et al., 2011). Walters et al. (2013) identified 1,219 AS events from 941 genes and Dou et al. (2015) identified AS events in two MADS-box genes. Some studies indicate that temperature can regulate $\mathrm{AS}$ and modulate the activity of splicing regulators and induce the AS of some genes in plants (Kwon et al., 2014; Capovilla et al., 2015). For example, at $31^{\circ} \mathrm{C}$, one isoform 
A

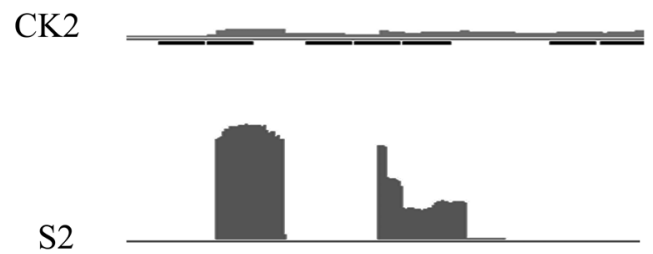

B

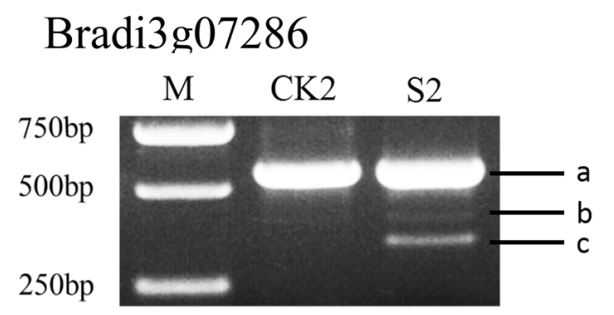

Actin

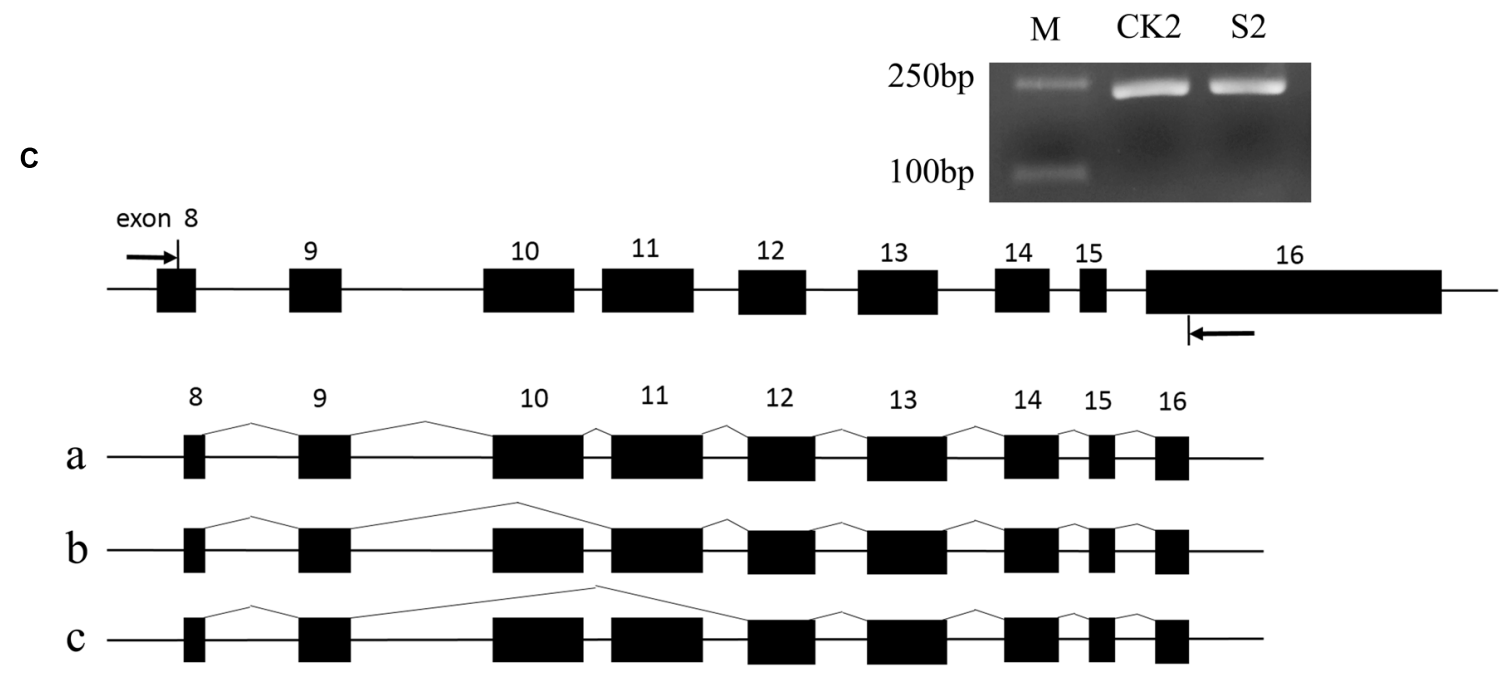

FIGURE 8 | Alternative splicing events in Bradi3g07286. (A) The IGV map of RNA-seq reds of CK2 and S2, respectively. (B) Result of semi-quantitative RT-PCR of $\mathrm{CK} 2$ and S2. (C) Diagram of three alternative splicing isoforms. Arrows in (C) indicate primers.

of the Arabidopsis gene HsfA2, HsfA2 II, was generated by splicing a 31-bp exon within one conserved intron. When the temperature rose to $42^{\circ} \mathrm{C}$, another isoform, HsfA2 III, was formed (Sugio et al., 2009). In rice, high temperature also induces AS. A temperature and drought-responsive gene, $D R E B 2 B$ generates a functional protein by AS. Under normal conditions, inclusion of the 53-bp exon 2 introduces a frame shift and premature termination, which leads to a nonfunctional transcript isoform (OsDREB2B1). Upon exposure to high temperatures, exon 2 is skipped and an ORF (Os-DREB2B2) only including exon 1 and 3 is formed (Matsukura et al., 2010).

In the present study, 46 DEGs associated with the spliceosome were identified in total. Of them, the expression of 43 DEGs was up-regulated under heat stress and 16 DEGs including three genes encoding splicing factor proteins were identified in our three sample groups. In particular, the expression level of one gene encoding one splicing factor in the Prp18 protein family was up-regulated 114.68611, 9,389.8651, and $1,894.9526$ times in the three treatment groups compared with the control. These results suggest that the ability for RNA splicing is enhanced at high temperatures. Likewise, 1,973 AS events distributed between 451 DEGs were identified (Supplementary Table S5) after heat treatment. This result clearly shows that high temperature induces AS by up-regulating the expression of genes involved in RNA splicing. In fact, plants can adjust the abundance of functional proteins through AS. Most likely some proteins are not necessary under normal conditions, but when the environmental conditions change, plants need some proteins to respond to the new conditions. In this case, the expression of genes relative to RNA processing and splicing are up-regulated and more AS events occur. Thus, heat stress not only regulates the expression of genes at the transcriptional level, but also at the post-transcriptional level. Of course in addition to AS, other manners of post-transcriptional regulation could include alternative protein folding and modification of proteins.

\section{The Molecular Mechanism in Response to Heat Stress Is Conserved}

In order to identify the responsive mechanism and commonalities in response to heat stress among different monocots, we compared our data with previous studies in rice (Zhang et al., 2012, 2013), wheat (Qin et al., 2008; Liu et al., 2015), maize (Fernandes et al., 2008) and switch grass (Li et al., 2013). In these species, protein folding, responses to abiotic stimulus, cellular and primary metabolic processes were significantly enriched BP identified in the associated GO terms. Furthermore, 
MF in binding associated chaperone and catalytic activity were enriched in all of the monocots tested, including B. distachyon.

In total, 885 and 395 homologous heat responsive genes from rice flag leaves and panicles were found in S2 and S3 samples of B. distachyon DEGs in this research, respectively.

To accommodate the imbalance and to survive in the extreme environments, plants need to reestablish their transcriptome, proteome, and metabolites (McClung and Davis, 2010; Mittler et al., 2012; Li et al., 2013). HSPs and other chaperones play a key role in protein-protein interactions such as folding, assisting in proper protein conformation, stabilizing partially unfolded proteins, and prevention of unwanted protein aggregation (Kotak et al., 2007; Li et al., 2013). To judge the conservation of molecular mechanisms in response to heat stress further, a comparison between our data (S2 and S3) and previous rice data (Zhang et al., 2012, 2013) was conducted. There were at least 83 genes encoding HSPs and other chaperones that were strongly induced by heat stress in $B$. distachyon (Supplementary Table S6), which is consistent with results that show that about $50 \%$ of homologous rice genes were also observed in heat-treated flag leaves or panicles (Supplementary Table S6).

The expression of many TF genes was altered by heat stress. In $B$. distachyon, 454 differentially expressed TFs were distributed in 49 families were identified. There were 13 families that had more than 15 genes in each; these families included ERF (9.25\%), bHLH (7.27\%), bZIP (7.27\%), MYB (6.83\%), NAC (6.17\%), and WRKY (6.17\%). Similarly, most of these $\mathrm{TF}$ families were the most significantly responsive $\mathrm{TF}$ genes observed in rice (Zhang et al., 2012, 2013) and wheat (Qin et al., 2008).

\section{REFERENCES}

Ashburner, M., Ball, C. A., Blake, J. A., Botstein, D., Butler, H., Cherry, J. M., et al. (2000). Gene ontology: tool for the unification of biology. The Gene Ontology Consortium. Nat. Genet. 25, 25-29. doi: 10.1038/ 75556

Asseng, S., Foster, I. A. N., and Turner, N. C. (2011). The impact of temperature variability on wheat yields. Global Change Biol. 17, 997-1012. doi: 10.1111/j. 1365-2486.2010.02262.x

Bita, C. E., and Gerats, T. (2013). Plant tolerance to high temperature in a changing environment: scientific fundamentals and production of heat stress-tolerant crops. Front. Plant Sci. 4:273. doi: 10.3389/fpls.2013.00273

Blum, A., Klueva, N., and Nguyen, H. T. (2001). Wheat cellular thermotolerance is related to yield under heat stress. Euphytica 117, 117-123. doi: 10.1023/A: 1004083305905

Cao, H., Xu, Y., Yuan, L., Bian, Y., Wang, L., Zhen, S., et al. (2016). Molecular characterization of the 14-3-3 gene family in Brachypodium distachyon L. Reveals High Evolutionary Conservation and Diverse Responses to Abiotic Stresses. Front. Plant Sci. 7:1099. doi: 10.3389/fpls.2016. 01099

Cao, J., Jiang, M., Li, P., and Chu, Z. (2016). Genome-wide identification and evolutionary analyses of the PP2C gene family with their expression profiling in response to multiple stresses in Brachypodium distachyon. BMC Genomics 17:175. doi: 10.1186/s12864-016-2526-4

Capovilla, G., Pajoro, A., Immink, R. G., and Schmid, M. (2015). Role of alternative pre-mRNA splicing in temperature signaling. Curr. Opin. Plant Biol. 27, 97-103. doi: $10.1016 /$ j.pbi.2015.06.016

\section{AUTHOR CONTRIBUTIONS}

Experimental design: HL; Experiments: SC and HL; Date analysis: HL and SC; Manuscript preparation: HL and SC; Supervision, fundings and reagents: HL.

\section{FUNDING}

This work was supported by the Natural Science Fundation of Xinjiang (No. 2016D01A052); the Open Project Program of State Key Laboratory of Crop Stress Biology for Arid Areas, NWAFU (No. CSBAA2016016); Research Funds from Xinjiang Agricultural Vocational Technical College(No. XJNZYKJ201501 and XJNZYKJ2015012); the Fundamental Research Funds for the Central Universities (No. Z109021565).

\section{ACKNOWLEDGMENTS}

We thank Professor Qixin Sun from Northwest A\&F University, for constructive advice on this project. We thank Dr. Xiaojun Nie from Northwest A\&F University, for asistance in the data submitting. We thank Shanghai NovelBio Company for the help with highthroughput sequencing related experiments.

\section{SUPPLEMENTARY MATERIAL}

The Supplementary Material for this article can be found online at: http://journal.frontiersin.org/article/10.3389/fpls.2016.02067/ full\#supplementary-material

Carmo-Silva, A. E., Gore, M. A., Andrade-Sanchez, P., French, A. N., Hunsaker, D. J., and Salvucci, M. E. (2012). Decreased CO2 availability and inactivation of Rubisco limit photosynthesis in cotton plants under heat and drought stress in the field. Environ. Exp. Bot. 83, 1-11. doi: 10.1016/j.envexpbot.2012.04.001

Cramer, G. R., Urano, K., Delrot, S., Pezzotti, M., and Shinozaki, K. (2011). Effects of abiotic stress on plants: a systems biology perspective. BMC Plant Biol. 11:163. doi: 10.1186/1471-2229-11-163

Deng, W. K., Wang, Y. B., Liu, Z. X., Cheng, H., and Xue, Y. (2014). HemI: a toolkit for illustrating heatmaps. PLoS ONE 9:11. doi: 10.1371/journal.pone.0111988

Ding, Y., Tao, Y., and Zhu, C. (2013). Emerging roles of microRNAs in the mediation of drought stress response in plants. J. Exp. Bot. 64, 3077-3086. doi: 10.1093/jxb/ert164

Dou, Y. H., Han, M. M., Sun, Q. X., and Hai-Feng, L. I. (2015). Alternative splicing and expression pattern analyses of two MADSBOX genes AGL6 and FUL1 in Brachypodium distachyon. J. Agric. Biotechnol. 23, 459-468.

Draper, J., Mur, L. A. J., Jenkins, G., Ghosh-Biswas, G. C., Bablak, P., Hasterok, R., et al. (2001). Brachypodium distachyon. A new model system for functional genomics in grasses. Plant Physiol. 127, 1539-1555. doi: 10.1104/pp.010196

Dupuy, D., Bertin, N., Hidalgo, C. A., Venkatesan, K., Tu, D., Lee, D., et al. (2007). Genome-scale analysis of in vivo spatiotemporal promoter activity in Caenorhabditis elegans. Nat. Biotechnol. 25, 663-668. doi: 10.1038/nbt1305

Fernandes, J., Morrow, D. J., Casati, P., and Walbot, V. (2008). Distinctive transcriptome responses to adverse environmental conditions in Zea mays L. Plant Biotechnol. J. 6, 782-798. doi: 10.1111/j.1467-7652.2008.00360.x

Frank, G., Pressman, E., Ophir, R., Althan, L., Shaked, R., Freedman, M., et al. (2009). Transcriptional profiling of maturing tomato (Solanum lycopersicum L.) microspores reveals the involvement of heat shock proteins, ROS scavengers, 
hormones, and sugars in the heat stress response. J. Exp. Bot. 60, 3891-3908. doi: $10.1093 / \mathrm{jxb} / \mathrm{erp} 234$

Guan, Q., Lu, X., Zeng, H., Zhang, Y., and Zhu, J. (2013). Heat stress induction of miR398 triggers a regulatory loop that is critical for thermotolerance in Arabidopsis. Plant J. 74, 840-851. doi: 10.1111/tpj.12169

Guo, A. Y., Chen, X., Gao, G., Zhang, H., Zhu, Q. H., Liu, X. C., et al. (2008). PlantTFDB: a comprehensive plant transcription factor database. Nucleic Acids Res. 36, D966-D969. doi: 10.1093/nar/gkm841

Harsant, J., Pavlovic, L., Chiu, G., Sultmanis, S., and Sage, T. L. (2013). High temperature stress and its effect on pollen development and morphological components of harvest index in the $\mathrm{C} 3$ model grass Brachypodium distachyon. J. Exp. Bot. 64, 2971-2983. doi: 10.1093/jxb/ert142

Hasanuzzaman, M., Nahar, K., Alam, M. M., Roychowdhury, R., and Fujita, M. (2013). Physiological, biochemical, and molecular mechanisms of heat stress tolerance in plants. Int. J. Mol. Sci. 14, 9643-9684. doi: 10.3390/ijms14059643

Hong, S. Y., Seo, P. J., Yang, M. S., Xiang, F., and Park, C. M. (2008). Exploring valid reference genes for gene expression studies in Brachypodium distachyon by real-time PCR. BMC Plant Biol. 8:112. doi: 10.1186/1471-2229-8-112

Huang, S., Liu, Z., Yao, R., Li, D., and Feng, H. (2015). Comparative transcriptome analysis of the petal degeneration mutant pdm in Chinese cabbage (Brassica campestris ssp. pekinensis) using RNA-Seq. Mol. Genet. Genomics 290, 1833-1847. doi: 10.1007/s00438-015-1041-7

International Brachypodium Initiative (2010). Genome sequencing and analysis of the model grass Brachypodium distachyon. Nature 463, 763-768. doi: 10.1038/ nature 08747

Jin, J., Zhang, H., Kong, L., Gao, G., and Luo, J. (2014). PlantTFDB 3.0: a portal for the functional and evolutionary study of plant transcription factors. Nucleic Acids Res. 42, 1182-1187. doi: 10.1093/nar/gkt1016

Kalra, S., Puniya, B. L., Kulshreshtha, D., Kumar, S., Kaur, J., Ramachandran, S., et al. (2013). De Novo transcriptome sequencing reveals important molecular networks and metabolic pathways of the plant, Chlorophytum borivilianum. PLoS ONE 8:e83336. doi: 10.1371/journal.pone.0083336

Kanehisa, M., Goto, S., Sato, Y., Furumichi, M., and Tanabe, M. (2012). KEGG for integration and interpretation of large-scale molecular data sets. Nucleic Acids Res. 40, D109-D114. doi: 10.1093/nar/gkr988

Kotak, S., Larkindale, J., Lee, U., von Koskull-Doring, P., Vierling, E., and Scharf, K. D. (2007). Complexity of the heat stress response in plants. Curr. Opin. Plant Biol. 10, 310-316. doi: 10.1016/j.pbi.2007.04.011

Kumar, R. R., Goswami, S., Sharma, S. K., Kala, Y. K., Rai, G. K., Mishra, D. C., et al. (2015a). Harnessing next generation sequencing in climate change: RNASeq analysis of heat stress-responsive genes in wheat (Triticum aestivum L.). OMICS 19, 632-647. doi: 10.1089/omi.2015.0097

Kumar, R. R., Pathak, H., Sharma, S. K., Kala, Y. K., Nirjal, M. K., Singh, G. P., et al. (2015b). Novel and conserved heat-responsive microRNAs in wheat (Triticum aestivum L.). Funct. Integr Genomics 15, 323-348. doi: 10.1007/s10142-0140421-0

Kwon, Y. J., Park, M. J., Kim, S. G., Baldwin, I. T., and Park, C. M. (2014). Alternative splicing and nonsense-mediated decay of circadian clock genes under environmental stress conditions in Arabidopsis. BMC Plant Biol. 14:136. doi: 10.1186/1471-2229-14-136

Labadorf, A., Link, A., Rogers, M. F., Thomas, J., Reddy, A. S., and Ben-Hur, A. (2010). Genome-wide analysis of alternative splicing in Chlamydomonas reinhardtii. BMC Genomics 11:114. doi: 10.1186/1471-2164$11-114$

Li, Y. F., Wang, Y. X., Tang, Y. H., Kakani, V. G., and Mahalingam, R. (2013). Transcriptome analysis of heat stress response in switchgrass (Panicum virgatum L.). BMC Plant Biol. 13:12. doi: 10.1186/1471-222913-153

Lindemose, S., O'Shea, C., Jensen, M. K., and Skriver, K. (2013). Structure, function and networks of transcription factors involved in abiotic stress responses. Int. J. Mol. Sci. 14, 5842-5878. doi: 10.3390/ijms14035842

Liu, G. T., Wang, J. F., Cramer, G., Dai, Z. W., Duan, W., Xu, H. G., et al. (2012). Transcriptomic analysis of grape (Vitis vinifera L.) leaves during and after recovery from heat stress. BMC Plant Biology 12:174. doi: 10.1186/1471-222912-174

Liu, M., Li, H., Su, Y., Li, W., and Shi, C. (2016). G1/ELE Functions in the development of rice lemmas in addition to determining identities of empty glumes. Front. Plant Sci. 7:1006. doi: 10.3389/fpls.2016.01006
Liu, X., and Chu, Z. (2015). Genome-wide evolutionary characterization and analysis of bZIP transcription factors and their expression profiles in response to multiple abiotic stresses in Brachypodium distachyon. BMC Genomics 16:227. doi: 10.1186/s12864-015-1457-9

Liu, Z., Xin, M., Qin, J., Peng, H., Ni, Z., Yao, Y., et al. (2015). Temporal transcriptome profiling reveals expression partitioning of homeologous genes contributing to heat and drought acclimation in wheat (Triticum aestivum $\mathrm{L}$.). BMC Plant Biol. 15:152. doi: 10.1186/s12870-015-0511-8

Livak, K. J., and Schmittgen, T. D. (2001). Analysis of relative gene expression data using real-time quantitative PCR and the 2(-Delta Delta C(T)) Method. Methods 25, 402-408. doi: 10.1006/meth.2001.1262

Macaulay, I. C., Haerty, W., Kumar, P., Li, Y. I., Hu, T. X., Teng, M. J., et al. (2015). G\&T-seq: parallel sequencing of single-cell genomes and transcriptomes. Nat. Methods 12, 519-522. doi: 10.1038/nmeth.3370

Mandadi, K. K., and Scholthof, K. B. (2015). Genome-wide analysis of alternative splicing landscapes modulated during plant-virus interactions in Brachypodium distachyon. Plant Cell 27, 71-85. doi: 10.1105/tpc.114.133991

Marquez, Y., Brown, J. W. S., Simpson, C., Barta, A., and Kalyna, M. (2012). Transcriptome survey reveals increased complexity of the alternative splicing landscape in Arabidopsis. Genome Res. 22, 1184-1195. doi: 10.1101/gr. 134106.111

Matsukura, S., Mizoi, J., Yoshida, T., Todaka, D., Ito, Y., Maruyama, K., et al. (2010). Comprehensive analysis of rice DREB2-type genes that encode transcription factors involved in the expression of abiotic stress-responsive genes. Mol. Genet. Genomics 283, 185-196. doi: 10.1007/s00438-009-0506-y

McClung, C. R., and Davis, S. J. (2010). Ambient thermometers in plants: from physiological outputs towards mechanisms of thermal sensing. Curr. Biol. 20, R1086-R1092. doi: 10.1016/j.cub.2010.10.035

Mishkind, M., Vermeer, J. E., Darwish, E., and Munnik, T. (2009). Heat stress activates phospholipase D and triggers PIP accumulation at the plasma membrane and nucleus. Plant J. 60, 10-21. doi: 10.1111/j.1365-313X.2009. 03933.x

Mittler, R. (2006). Abiotic stress, the field environment and stress combination. Trends Plant Sci. 11, 15-19. doi: 10.1016/j.tplants.2005.11.002

Mittler, R., Finka, A., and Goloubinoff, P. (2012). How do plants feel the heat? Trends Biochem. Sci. 37, 118-125. doi: 10.1016/j.tibs.2011.11.007

Narayanan, S., Tamura, P. J., Roth, M. R., Prasad, P. V., and Welti, R. (2016). Wheat leaf lipids during heat stress: I. High day and night temperatures result in major lipid alterations. Plant Cell Environ. 39, 787-803. doi: 10.1111/pce. 12649

Prasad, P. V. V., Pisipati, S. R., Momcilovic, I., and Ristic, Z. (2011). Independent and combined effects of high temperature and drought stress during grain filling on plant yield and chloroplast EF-Tu expression in spring wheat. J. Agron. Crop Sci. 197, 430-441. doi: 10.1111/j.1439-037X.2011.00477.x

Priest, H. D., Fox, S. E., Rowley, E. R., Murray, J. R., Michael, T. P., and Mockler, T. C. (2014). Analysis of global gene expression in Brachypodium distachyon reveals extensive network plasticity in response to abiotic stress. PLoS ONE 9:e87499. doi: 10.1371/journal.pone.0087499

Qin, D., Wang, F., Geng, X., Zhang, L., Yao, Y., Ni, Z., et al. (2015). Overexpression of heat stress-responsive TaMBF1c, a wheat (Triticum aestivum L.) multiprotein bridging factor, confers heat tolerance in both yeast and rice. Plant Mol. Biol. 87, 31-45. doi: 10.1007/s11103-014-0259-9

Qin, D., Wu, H., Peng, H., Yao, Y., Ni, Z., Li, Z., et al. (2008). Heat stressresponsive transcriptome analysis in heat susceptible and tolerant wheat (Triticum aestivum L.) by using wheat genome array. BMC Genomics 9:432. doi: 10.1186/1471-2164-9-432

Reon, B. J., and Dutta, A. (2016). Biological processes discovered by highthroughput sequencing. Am. J. Pathol. 186, 722-732. doi: 10.1016/j.ajpath.2015. 10.033

Rizhsky, L., Hallak-Herr, E., Van Breusegem, F., Rachmilevitch, S., Barr, J. E., Rodermel, S., et al. (2002). Double antisense plants lacking ascorbate peroxidase and catalase are less sensitive to oxidative stress than single antisense plants lacking ascorbate peroxidase or catalase. Plant J. 32, 329-342. doi: 10.1046/j. 1365-313X.2002.01427.x

Rizhsky, L., Liang, H., Shuman, J., Shulaev, V., Davletova, S., and Mittler, R. (2004). When defense pathways collide. The response of Arabidopsis to a combination of drought and heat stress. Plant Physiol. 134, 1683-1696. doi: 10.1104/pp.103. 033431 
Rodriguez, V. M., Soengas, P., Alonso-Villaverde, V., Sotelo, T., Cartea, M. E., and Velasco, P. (2015). Effect of temperature stress on the early vegetative development of Brassica oleracea L. BMC Plant Biol. 15:145. doi: 10.1186/ s12870-015-0535-0

Rollins, J. A., Habte, E., Templer, S. E., Colby, T., Schmidt, J., and von Korff, M. (2013). Leaf proteome alterations in the context of physiological and morphological responses to drought and heat stress in barley (Hordeum vulgare L.). J Exp Bot 64, 3201-3212. doi: 10.1093/jxb/ert158

Sablok, G., Gupta, P. K., Baek, J. M., Vazquez, F., and Min, X. J. (2011). Genomewide survey of alternative splicing in the grass Brachypodium distachyon: a emerging model biosystem for plant functional genomics. Biotechnol. Lett. 33, 629-636. doi: 10.1007/s10529-010-0475-6

Salvucci, M. E., and Crafts-Brandner, S. J. (2004a). Mechanism for deactivation of Rubisco under moderate heat stress. Physiol. Plant. 122, 513-519. doi: 10.1111/ j.1399-3054.2004.00419.x

Salvucci, M. E., and Crafts-Brandner, S. J. (2004b). Relationship between the heat tolerance of photosynthesis and the thermal stability of rubisco activase in plants from contrasting thermal environments. Plant Physiol. 134, 1460-1470. doi: 10.1104/pp.103.038323

Song, Y. P., Chen, Q. Q., Ci, D., Shao, X. N., and Zhang, D. Q. (2014). Effects of high temperature on photosynthesis and related gene expression in poplar. BMC Plant Biol. 14:111. doi: 10.1186/1471-2229-14-111

Staiger, D., and Brown, J. W. (2013). Alternative splicing at the intersection of biological timing, development, and stress responses. Plant Cell 25, 3640-3656. doi: 10.1105/tpc.113.113803

Stamm, S., Ben-Ari, S., Rafalska, I., Tang, Y., Zhang, Z., Toiber, D., et al. (2005). Function of alternative splicing. Gene 344, 1-20. doi: 10.1016/j.gene.2004. 10.022

Stratonovitch, P., and Semenov, M. A. (2015). Heat tolerance around flowering in wheat identified as a key trait for increased yield potential in Europe under climate change. J. Exp. Bot. 66, 3599-3609. doi: 10.1093/jxb/ erv070

Subburaj, S., Luo, N., Lu, X., Li, X., Cao, H., Hu, Y., et al. (2015). Molecular characterization and evolutionary origins of farinin genes in Brachypodium distachyon L. J. Appl. Genet. 57, 287-303. doi: 10.1007/s13353-015-0316-3

Sugio, A., Dreos, R., Aparicio, F., and Maule, A. J. (2009). The cytosolic protein response as a subcomponent of the wider heat shock response in Arabidopsis. Plant Cell 21, 642-654. doi: 10.1105/tpc.108.062596

Suzuki, N., Rivero, R. M., Shulaev, V., Blumwald, E., and Mittler, R. (2014). Abiotic and biotic stress combinations. New Phytol 203, 32-43. doi: 10.1111/nph.12797

Vile, D., Pervent, M., Belluau, M., Vasseur, F., Bresson, J., Muller, B., et al. (2012). Arabidopsis growth under prolonged high temperature and water deficit: independent or interactive effects? Plant Cell Environ. 35, 702-718. doi: 10.1111/j.1365-3040.2011.02445.x

Walters, B., Lum, G., Sablok, G., and Min, X. J. (2013). Genome-wide landscape of alternative splicing events in Brachypodium distachyon. DNA Res. 20, 163-171. doi: 10.1093/dnares/dss041

Wang, K., Singh, D., Zeng, Z., Coleman, S. J., Huang, Y., Savich, G. L., et al. (2010). MapSplice: accurate mapping of RNA-seq reads for splice junction discovery. Nucleic Acids Res. 38, e178. doi: 10.1093/nar/gkq622

Wang, L., Feng, Z., Wang, X., Wang, X., and Zhang, X. (2010). DEGseq: an $\mathrm{R}$ package for identifying differentially expressed genes from RNA-seq data. Bioinformatics 26, 136-138. doi: 10.1093/bioinformatics/btp612
Way, D. A., and Oren, R. (2010). Differential responses to changes in growth temperature between trees from different functional groups and biomes: a review and synthesis of data. Tree Physiol. 30, 669-688. doi: 10.1093/treephys/ tpq015

Westgate, M. E. (1994). Water status and development of the maize endosperm and embryo during drought. Crop Sci. 34, 76-83. doi: 10.2135/cropsci1994. 0011183X003400010014x

Wu, L., Taohua, Z., Gui, W., Xu, L., Li, J., and Ding, Y. (2015). Five pectinase gene expressions highly responding to heat stress in rice floral organs revealed by RNA-seq analysis. Biochem. Biophys. Res. Commun. 463, 407-413. doi: 10.1016/ j.bbrc.2015.05.085

Xin, M., Wang, Y., Yao, Y., Xie, C., Peng, H., Ni, Z., et al. (2010). Diverse set of microRNAs are responsive to powdery mildew infection and heat stress in wheat (Triticum aestivum L.). BMC Plant Biol. 10:123. doi: 10.1186/1471-2229$10-123$

Xue, G. P., Drenth, J., and McIntyre, C. L. (2015). TaHsfA6f is a transcriptional activator that regulates a suite of heat stress protection genes in wheat (Triticum aestivum L.) including previously unknown Hsf targets. J. Exp. Bot. 66, 1025-1039. doi: 10.1093/jxb/eru462

You, J., Zhang, L., Song, B., Qi, X., and Chan, Z. (2015). Systematic analysis and identification of stress-responsive genes of the NAC gene family in Brachypodium distachyon. PLOS ONE 10:e0122027. doi: 10.1371/journal.pone. 0122027

Young, L. W., Wilen, R. W., and Bonham-Smith, P. C. (2004). High temperature stress of Brassica napus during flowering reduces micro- and megagametophyte fertility, induces fruit abortion, and disrupts seed production. J. Exp. Bot. 55, 485-495. doi: 10.1093/jxb/erh038

Zhang, L., You, J., and Chan, Z. (2015). Identification and characterization of TIFY family genes in Brachypodium distachyon. J Plant Res. 128, 995-1005. doi: 10.1007/s10265-015-0755-2

Zhang, X., Li, J., Liu, A., Zou, J., Zhou, X., Xiang, J., et al. (2012). Expression profile in rice panicle: insights into heat response mechanism at reproductive stage. PLoS ONE 7:e49652. doi: 10.1371/journal.pone.0049652.g001

Zhang, X., Rerksiri, W., Liu, A., Zhou, X., Xiong, H., Xiang, J., et al. (2013). Transcriptome profile reveals heat response mechanism at molecular and metabolic levels in rice flag leaf. Gene 530, 185-192. doi: 10.1016/j.gene.2013. 08.048

Zhou, X., Wu, W., Li, H., Cheng, Y., Wei, N., Zong, J., et al. (2014). Transcriptome analysis of alternative splicing events regulated by SRSF10 reveals positiondependent splicing modulation. Nucleic Acids Res. 42, 4019-4030. doi: 10.1093/ nar/gkt1387

Conflict of Interest Statement: The authors declare that the research was conducted in the absence of any commercial or financial relationships that could be construed as a potential conflict of interest.

Copyright (C) 2017 Chen and Li. This is an open-access article distributed under the terms of the Creative Commons Attribution License (CC BY). The use, distribution or reproduction in other forums is permitted, provided the original author(s) or licensor are credited and that the original publication in this journal is cited, in accordance with accepted academic practice. No use, distribution or reproduction is permitted which does not comply with these terms. 